\title{
Microstructure and Hardness of Surface Melting Hardened Zone of Mold Steel, SM45C using Yb:YAG Disk Laser
}

\author{
Kwang-Hyeon Lee*, Seong-Won Choi**, Tae-Jin Yoon ${ }^{* * *}$ and Chung-Yun Kang**, $\dagger$ \\ *Korea Institute of Machinery \& Materials, Busan Laser Application Support Center, Busan 46747, Korea \\ **Dept. of Material Science and Engineering, Pusan National University, Busan 46239, Korea \\ ***National core research center, Pusan National University, Busan 46239, Korea
}

†Corresponding author : kangcy@pusan.ac.kr

(Received February 3, 2016 ; Revised February 15, 2016 ; Accepted February 18, 2016)

\begin{abstract}
This study applied laser surface melting process using CW(Continuous wave) Yb:YAG laser and cold-work die steel SM45C and investigated microstructure and hardness. Laser beam speed, power and beam interval are fixed at $70 \mathrm{~mm} / \mathrm{sec}, 2.8 \mathrm{~kW}$ and $800 \mu \mathrm{m}$ respectively. Depth of Hardening layer(Melting zone) was a minimum of $0.8 \mathrm{~mm}$ and a maximum of $1.0 \mathrm{~mm}$ that exceeds the limit of minimum depth $0.5 \mathrm{~mm}$ applying trimming die. In all weld zone, macrostructure was dendrite structure. At the dendrite boundary, $\mathrm{Mn}, \mathrm{Al}, \mathrm{S}$ and $\mathrm{O}$ was segregated and $\mathrm{MnS}$ and $\mathrm{Al}$ oxide existed. However, this inclusion didn't observe in the heat-affected zone (HAZ). As a result of interpreting phase transformation of binary diagram, $\mathrm{MnS}$ crystallizes from liquid. Also, it estimated that $\mathrm{Al}$ oxide forms by reacting with oxygen in the atmosphere. The hardness of the melting zone was from $650 \mathrm{Hv}$ to $660 \mathrm{Hv}$ regardless of the location that higher $60 \mathrm{Hv}$ than the hardness of the HAZ that had maximum $600 \mathrm{Hv}$. In comparison with the size of microstructure using electron backscatter diffraction(EBSD), the size of microstructure in the melting zone was smaller than HAZ. Because it estimated that cooling rate of laser surface melting process is faster than water quenching.
\end{abstract}

Key Words : Laser hardening, Yb:YAG Disc laser, SM45C, Laser surface melting, Trimming die

\section{Introduction}

SM45C, medium carbon steel, is widely used in industrial field for the production of machine parts such as crank and gear as well as the material of cold-work die steel. However, there can be damage such as abrasion easily when it is used for forming or cutting of advanced high strength steels which is currently increasing in use though it is used after surface hardening process on the material through surface preparation such as heat treatment ${ }^{1,2}$. To improve this problem, surface modification such as chemical vapor deposition (CVD), physical vapor deposition (PVD) has been applied ${ }^{3-5)}$, however, the separation phenomenon has been frequently reported due to thin hardened layer depth and brittleness ${ }^{6}$.

This problem can be improved by increasing the hardened layer depth and combine the base material and hardened layer closely ${ }^{7}$. Laser surface melting hardening technology is a surface modification technology of material using rapid heating and cooling feature of laser. Especially, laser is proper for surface hardening of mold material because the hardened layer can be formed deeply, no limitation in material shape, and selective laser surface treatment is possible on the part need to be hardened. Based on these technical features, several researches for laser surface melting hardening have been reported currently ${ }^{8-10)}$. However, Shin et al., reported that the hardened layer is about $300 \mu \mathrm{m}$ in surface hardening using Nd:YAG laser ${ }^{11)}$. Choi et al., reported that enough hardened layer depth $(400 \sim 500 \mu \mathrm{m})$ cannot be assured when hardening by diode laser ${ }^{7)}$.

It is reported that $\mathrm{Mo}, \mathrm{Cr}, \mathrm{V}$, and $\mathrm{C}$ which are included in the steel can form precipitation and attain grain refining by heat treatment. Additionally strength, hardness, abrasion resistance, and corrosion resistance are improved by forming microstructure such as martensite 
which is high in hardness by laser surface hardness ${ }^{1)}$. However, in case of SM45C, it is difficult to expect the surface hardening by carbide forming because there is few carbide forming elements in it. Alain Kusmoko et al,. reported that the reason of hardening heat treatment of SM45C is the phase transformation of martensite ${ }^{12}$. Especially, in case of melting hardening using laser heat source, researchers reported that the mechanical property and hardened layer depth are different with the microstructure of hardened layer formed by existing heat treatment process due to the repetition of extreme rapid heating and cooling ${ }^{13)}$. Although the SM45C of die steel is used in many cases, the study on the mechanism for laser surface melting hardening is nothing at all. Therefore, in this research, systematically study the correlation between the change in microstructure of surface melted part and heat-affected zone and hardness on the SM45C, medium carbon steel, on laser surface melting hardening process with minimum over $600 \mu \mathrm{m}$ hardness depth using Yb:YAG high power disc laser.

\section{Experimental Procedures}

The chemical composition of SM45C steel used in this research is shown in Table 1, and the specimen thickness is $15 \mathrm{~mm}$. The surfaces of the specimen were mechanically worked as even and flat. The laser used for laser melting hardening treatment was Yb:YAG disc laser and the maximum output is $8 \mathrm{~kW}$ (Trumpf Tru Disk8002, Busan Laser Technology Support Center, Korea Institute of Machinery \& Materials). The optical system of $0.4 \mathrm{~mm}$ beam diameter was used and being installed on 6-axes laser precision processing robot. Laser melting hardening treatment condition are fixed as laser focusing size of $400 \mu \mathrm{m}$, laser processing speed of $70 \mathrm{~mm} / \mathrm{s}$, beam interval of $800 \mu \mathrm{m}$, and laser power of $2.8 \mathrm{~kW}$. Ar gas $(8 \mathrm{~L} / \mathrm{min})$ was supplied as shielding gas and the laser surface melting hardening treatment was performed as zigzag direction as shown in Fig. 1(a).

The microstructure of hardened layer was observed with optical microscope and scanning electron microscope(SEM) by revealing the structure using viela solution $\left\{\mathrm{CH}_{3} \mathrm{OH}(100 \mathrm{~mL})+\mathrm{HCl}(4 \mathrm{~mL})+\right.$ Picric $\left.(1 \mathrm{~g})\right\}$ after grind the specimen. To analyze composition profile in melted zone, field emission electron probe micro analyzer (FE-EPMA) zone analysis was used and EBSD was used in comparison of melted zone and

Table 1 Chemical composition of SM45C

\begin{tabular}{|c|c|c|c|c|c|c|c|c|c|c|c|}
\hline \multirow{3}{*}{ SM45C } & \multicolumn{10}{|c|}{ Chemical composition (wt.\%) } \\
\cline { 2 - 11 } & $\mathrm{C}$ & $\mathrm{Mn}$ & $\mathrm{Si}$ & $\mathrm{Cr}$ & $\mathrm{Ni}$ & $\mathrm{Mo}$ & $\mathrm{V}$ & $\mathrm{W}$ & $\mathrm{P}$ & $\mathrm{S}$ & $\mathrm{Fe}$ \\
\cline { 2 - 11 } & 0.41 & 0.70 & 0.25 & 0.02 & 0.03 & - & - & - & 0.03 & 0.05 & Bal. \\
\hline
\end{tabular}

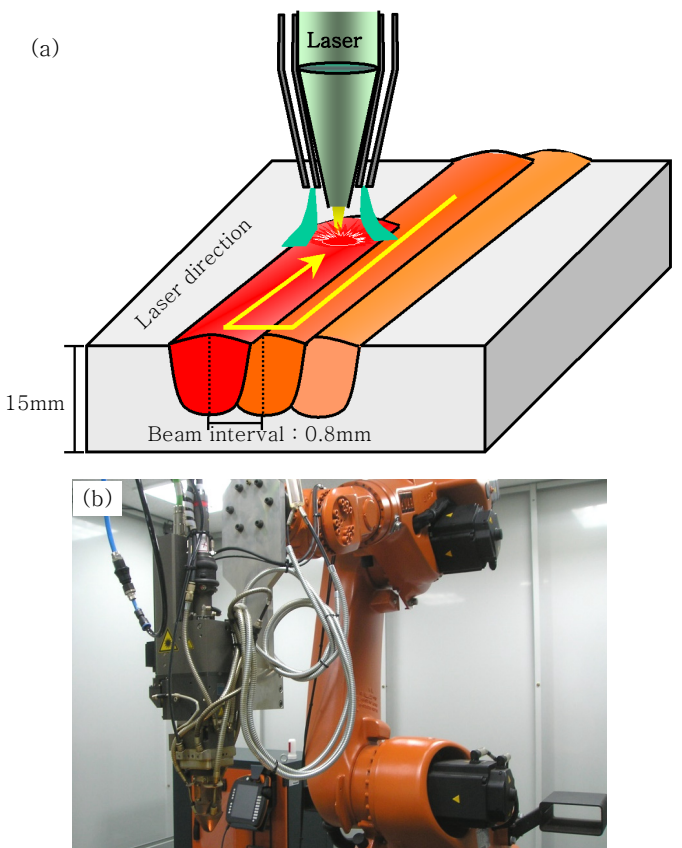

Fig. 1 Schematic illustration of laser hardening (a), Experimental equipment of laser surface melted hardening (b)

heat-affected zone grain size. To investigate the phase transformation on each zone, Thermo - Calc. ${ }^{\mathrm{TM}}$ (TCW) was used. The hardness of melted zone, heat-affected zone and the base material was measured using micro vickers hardness with $300 \mathrm{kgf}$ load and time duration 10 seconds.

\section{Result and Discussion}

\subsection{Hardness Distribution and Micro Structure of Surface Melted Hardened Zone}

Fig. 2 is surface melted bead, and left side is finally formed bead. Average bead maximum depth of penetration is $1.0 \mathrm{~mm}$ and minimum is $0.8 \mathrm{~mm}$, and the width of bead is $1.1 \mathrm{~mm}$.

Surface melted hardened layer is formed by bead over-

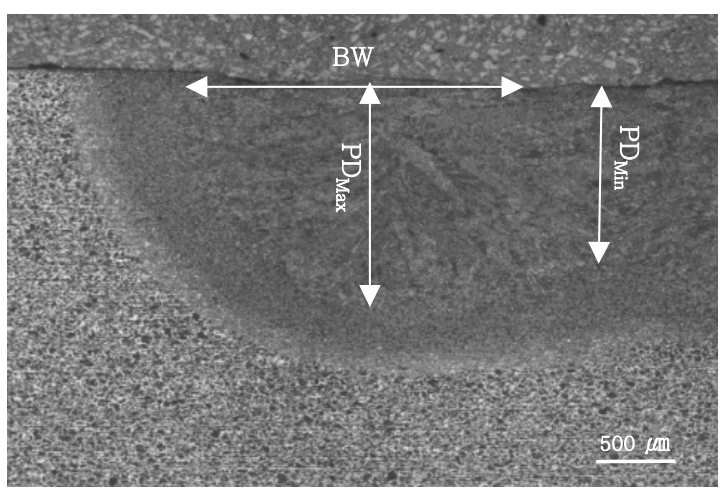

Fig. 2 Cross-section of laser surface melting layer 


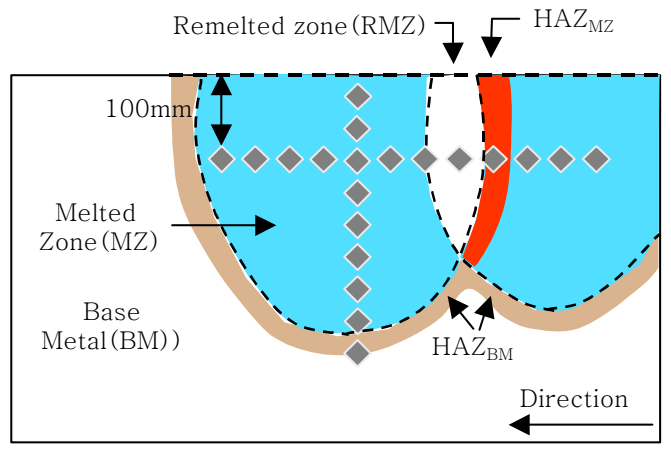

Fig. 3 Schematic illustration of lase surface melting layer

lapped with $0.8 \mathrm{~mm}$ distance, so preceding bead is partially remelted by following laser beam, and forms heat-affected zone by the heat effect. Meted zone can be divided as melted zone (MZ) formed by following laser beam, remelted zone (RMZ) formed by remelting of $M Z$ by preceding laser beam, heat affected zone in $M Z$ $\left(\mathrm{HAZ}_{\mathrm{MZ}}\right.$ ) formed by heat effect, and heat affected zone in base metal $\left(\mathrm{HAZ}_{\mathrm{BM}}\right)$ formed on base material as shown in Fig. 3. Each zone has different forming procedure, so the hardness of each zone expected as different, therefore, vickers hardness were laterally measured with $100 \mu \mathrm{m}$ distance, and also measured with $100 \mu \mathrm{m}$ distance to depth direction to include $\mathrm{MZ}, \mathrm{RMZ}$, and HAZ at $100 \mu \mathrm{m}$ under the surface as shown in Fig. 3. Fig. 4 shows the hardness distribution of each lateral (a) and depth direction (b). The lateral hardness distribution shows about \pm 4 hardness value deviation according to the location, and it means there is almost no difference. Also, the hardness value difference for depth direction according to the location in MZ has less difference; however, it is rapidly decreased from HAZ to BM.

Microstructure in $\mathrm{MZ}, \mathrm{RMZ}$, and $\mathrm{HAZ}_{\mathrm{MZ}}$ in melted zone were compared to understand the reason of almost same hardness value for each area in MZ and the results were shown in Fig. 5. Fig. 5(a), (b), and (c) shows the microstructure observed by optical microscope for each $\mathrm{MZ}, \mathrm{RMZ}$, and $\mathrm{HAZ}_{\mathrm{MZ}}$, and Fig. 5(d), (e), and (f) shows the magnified microstructure marked as $\square$ for (a), (b), and (c) by SEM. Fig. 6 is the solidification microstructure in MZ observed by OM. Based on the comparison between Fig. 5 and Fig. 6, black band type part in Fig. 5 can be identified as dendrite boundary. In the microstructure from OM and SEM in Fig. 5, the difference according to the zone in MZ.

Fig. 7(a) and (b) shows the microstructure in MZ by $\mathrm{OM}$ and SEM, and Fig. 7(c) to (h) reveals the result of elemental mapping analysis by EPMA corresponding Fig. 7(b). Based on it, it is indicated that the band zone
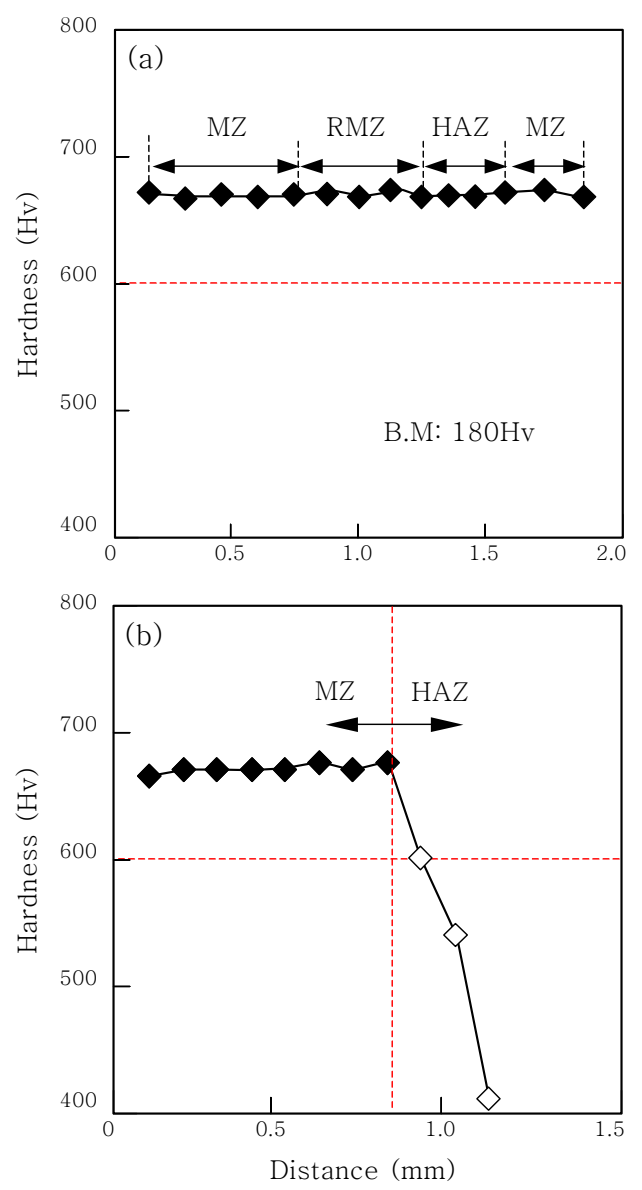

Fig. 4 Hardness distributions of width(a) and depth(b) direction in the melting zone
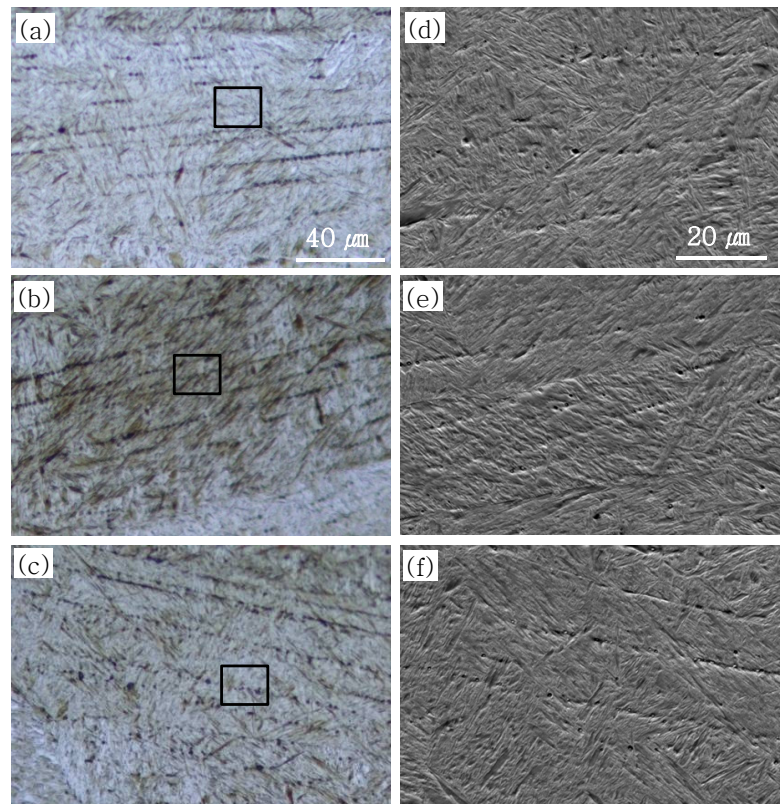

Fig. 5 Optical microstructures and SEM structures of laser surface melting layer (a), (d) melted zone, (b),(e) remelted zone, (c), (f) HAZ in previous melted zone 


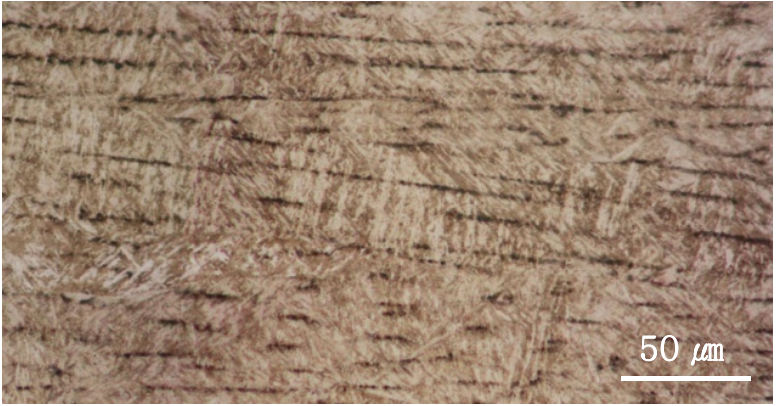

Fig. 6 Optical microstructures of laser surface melted zone
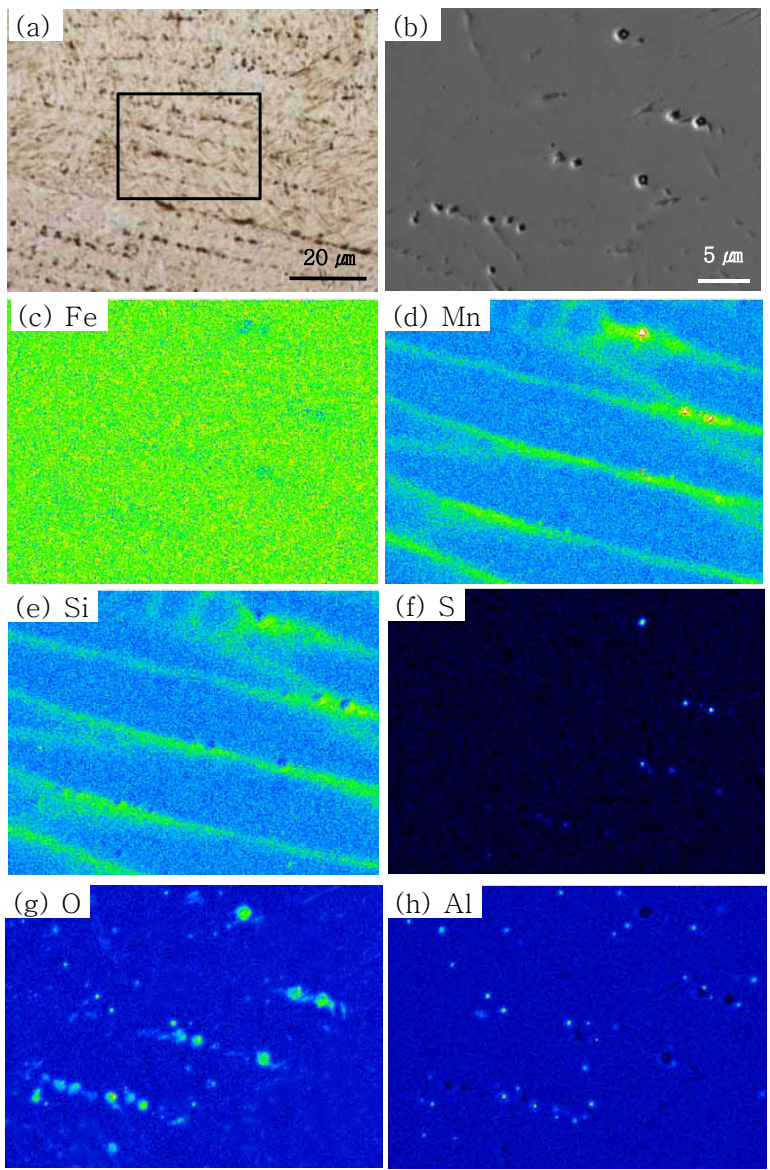

Fig. 7 Optical(a), SEM(b) microstructure and EPMA mapping results showing distribution of elements in melted zone

in microstructure segregates $\mathrm{Mn}$ and $\mathrm{Si}$, and concentrated $\mathrm{Al}, \mathrm{S}$, and $\mathrm{O}$ shaped spheral type are existed in it. That is, MnS and Al based oxide are existed in it.

Fig. 8 shows the microstructure of BM by SEM, and it shows that BM has layer structure with ferrite and pearlite and no inclusion is existed.

Therefore, the segregation layer formed on surface $\mathrm{MZ}$ and the band zone with $\mathrm{MnS}, \mathrm{Al}$ based oxide were expected forming during the solidification after melting.

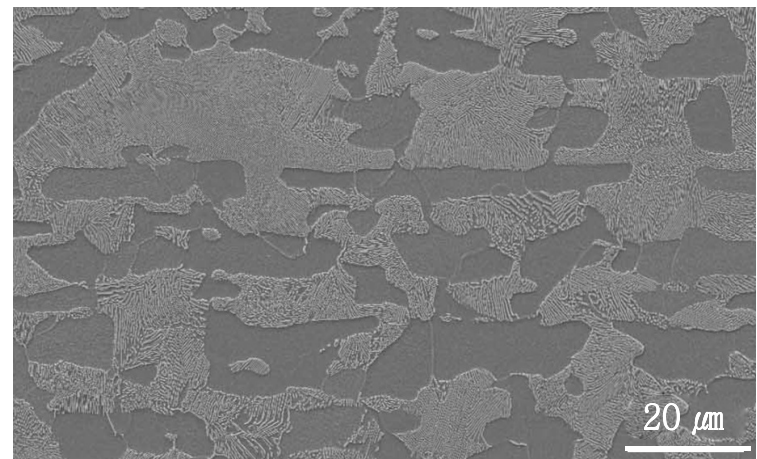

Fig. 8 SEM microstructure of the base metal

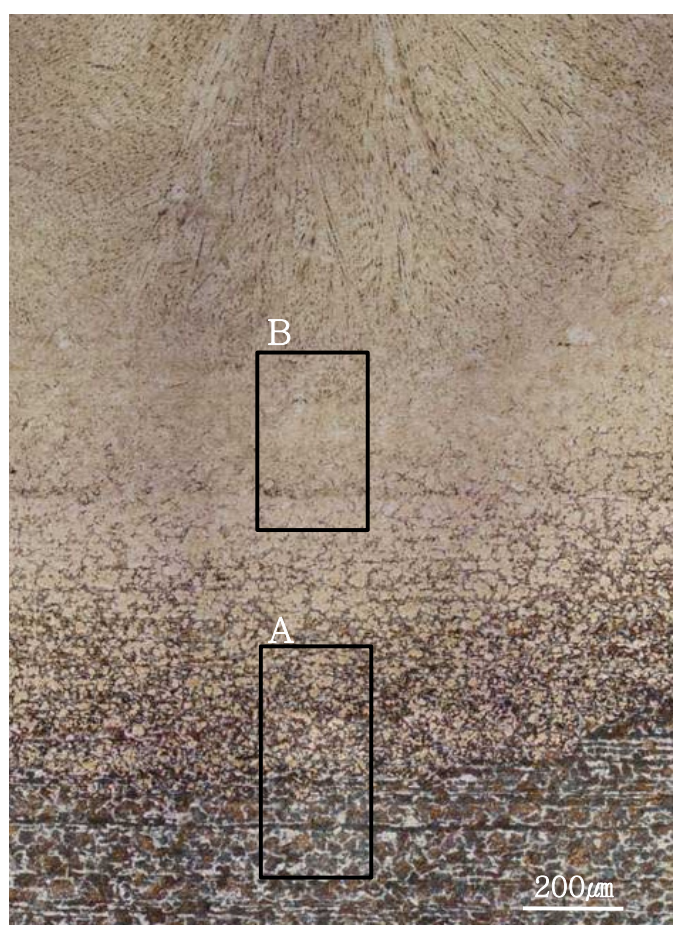

Fig. 9 Optical microstructure between the melted zone and the base metal

\subsection{Hardness and .micro structure between melted zone and base material}

Fig. 9 shows the macrostructure between $\mathrm{MZ}$ and $\mathrm{BM}$ observed by OM. By this macrostructure, the microstructure cannot be classified, so the BM/HAZ interface part and HAZ/MZ interface part were magnified as indicated as $\mathrm{A}$ and $\mathrm{B}$ in the figure. Fig. 10(a) is the magnified microstructure of $\mathrm{BM} / \mathrm{HAZ}$ interface part by SEM, and Fig. 10(b) to (e) are the magnified microstructure of $\mathrm{BM}, \mathrm{H}_{1}-\mathrm{H}_{3}$ indicated in Fig. 10(a) by SEM. The proeutectoid ferrite $(\mathrm{F})$ is decreased from $\mathrm{BM}$ to $\mathrm{MZ}$ in Fig. 10(a). Also, by the comparison of Fig. 10(b) to (e), from $\mathrm{BM}$ (b) to $\mathrm{H}_{3}(\mathrm{e})$, the lamella structure in pearlite gradually disappears and changes to new nee- 


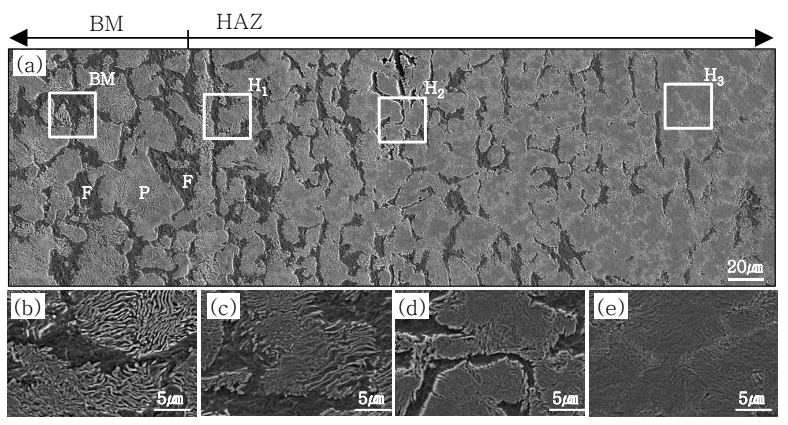

Fig. 10 (a)SEM structures from the base metal to HAZ, (b)-(e) enlarged SEM structures of area denoted as $\mathrm{BM}, \mathrm{H}_{1}, \mathrm{H}_{2}, \mathrm{H}_{3}$ in (a)

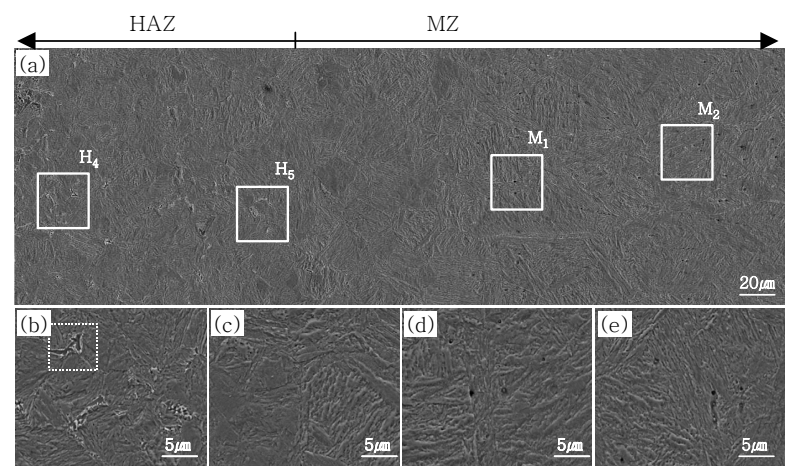

Fig. 11 (a)SEM structures from HAZ to melted zone, (b)-(e) enlarged SEM structures of area denoted as $\mathrm{H}_{4}, \mathrm{H}_{5}, \mathrm{M}_{1}, \mathrm{M}_{2}$ in (a)

dlelike structure, therefore, ferrite is not exist and almost needlelike structure at $\mathrm{H}_{4}(\mathrm{e})$. Fig. 11(a) is magnified microstructure of HAZ/MZ (B) interface part by SEM, and Fig. 11(b) to (e) are magnified microstructure of $\mathrm{H}_{4}-\mathrm{M}_{2}$ indicated in (a) by SEM. Based on the comparison between Fig. 10(a) and Fig. 11(a), ferrite is almost not shown, and lamella structure perlite is not observed. In the comparison of HAZ ((b), (c)) and MZ (d), there are MnS and Al based oxide in MZ, but not existed in HAZ. Based on it, it is identified that inclusions are formed during the solidification. Table 2 shows $\mathrm{BM}$ to $\mathrm{M}_{2}$ in Fig. 10 .

The needlelike structure in HAZ and MZ is martensite, and it seems that the reason of increasing hardness from $\mathrm{BM}$ to $\mathrm{MZ}$ is that the ferrite is disappeared and pearlite transformed to martensite.

Especially, HAZ (melted zone boundary) and MZ is consisted with martensite completely, but the hardness in $\mathrm{MZ}$ is higher over about $60 \mathrm{Hv}$. It is expected by the difference of martensite, so compared the martensite size using EBSD. Fig. 12 is the analyzed result of $\mathrm{H}_{5}$ and $\mathrm{M}_{1}$ zone using EBSD in Fig. 11.

Fig. 11(a) and (b) is image quality (IQ) map showing crystal grain classified with average mean strength by Kikuchi band, and Fig. 11(c) and (d) is inverse pole fig-

Table 2 Hardness near area denoted as $\mathrm{x}$ in (a) Fig. 10 and Fig. 11

\begin{tabular}{|c|c|c|c|c|c|c|c|c|c|}
\hline Area & $\mathrm{BM}$ & $\mathrm{H}_{1}$ & $\mathrm{H}_{2}$ & $\mathrm{H}_{3}$ & $\mathrm{H}_{4}$ & $\mathrm{H}_{5}$ & $\mathrm{M}_{1}$ & $\mathrm{M}_{2}$ & W.Q \\
\hline $\mathrm{Hv}$ & $\begin{array}{c}180 \sim \\
200\end{array}$ & $\begin{array}{c}280 \sim \\
300\end{array}$ & $\begin{array}{c}350 \sim \\
400\end{array}$ & $\begin{array}{c}450 \sim \\
510\end{array}$ & $\begin{array}{c}570 \sim \\
590\end{array}$ & $\begin{array}{c}590 \sim \\
600\end{array}$ & $\begin{array}{c}590 \sim \\
600\end{array}$ & $\begin{array}{c}650 \sim \\
660\end{array}$ & 580 \\
\hline
\end{tabular}
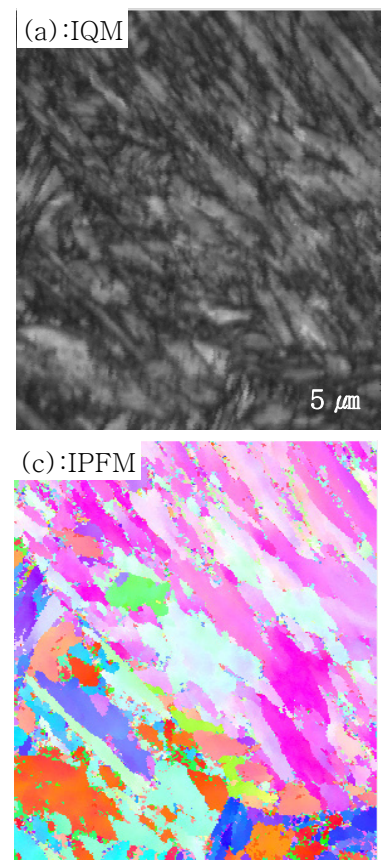

Fig. 12 Results obtained by EBSD. (a),(b) image quality map, (c), (d) inverse pole figure map. (a), (c) HAZ, (b), (d) MZ

ure (IPF) map divided with color for crystal orientation of crystal grain. Fig. 11(a) and (c) have been resulted from $\mathrm{H}_{5}$, and Fig. 11(b) and (d) are from $\mathrm{M}_{1}$. From this, the hardness in fusion zone (FZ) is higher than that of HAZ because martensite size of FZ is much refined. However, the austenite in FZ is more refiner because the austenite in HAZ is created and grown by reheating. On the other hand, austenite in FZ is formed during the solidification by rapid cooling. Therefore, the cooling speed is same, so it is considered that the martensite size is refined because the austenite in FZ is refined.

\subsection{Phase transformation of surface melted hard- ened zone}

To understand the phase transformation process of $\mathrm{MZ}$ inside and BM/HAZ boundary during the melting and cooling process by laser beam, binary system constitutional diagram is prepared using Thermo-Calc. ${ }^{\mathrm{TM}}$ (TCW5) software.

Fig. 13 is binary system constitutional diagram of $\mathrm{Fe}$ (0.70 Mn- 0.28 Si- 0.02 Cr- 0.04 Al- 0.05 S) - C, and 


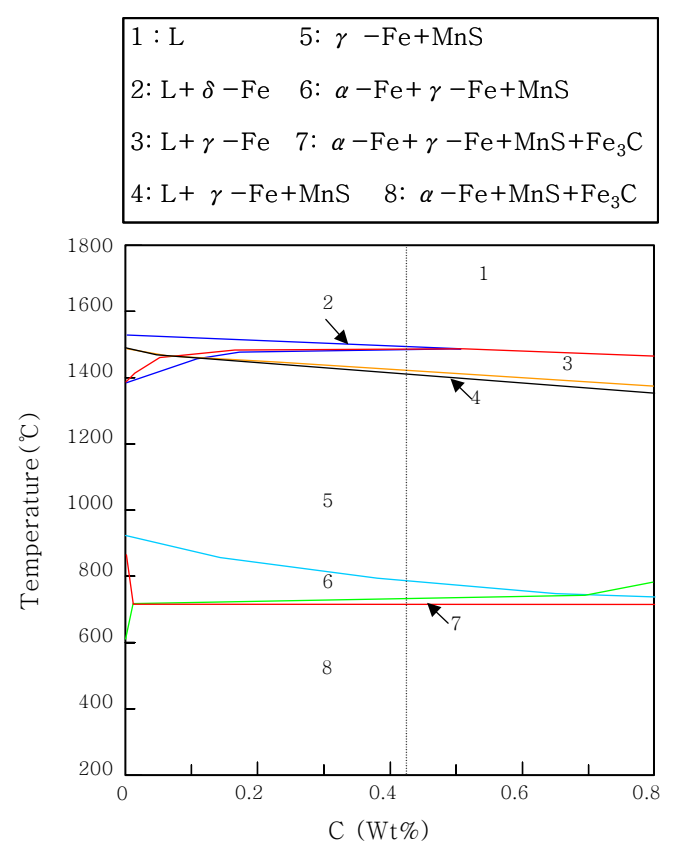

Fig. 13 Quasi-binary phase diagram for calculated using Thermo-Calc ${ }^{\mathrm{TM}}$ software

the figures for the marked zone numbers were expressed on the constitutional diagram. The formation of dendrite cores occurs because the almost austenite $(\mathrm{\gamma})$ formed by faster cooling speed during solidification of liquid phase of $\mathrm{BM}(0.41 \mathrm{wt} \% \mathrm{C})$. As the solidification is processed, $\mathrm{Mn}, \mathrm{Si}$, and $\mathrm{Al}$ are segregated, and $\mathrm{MnS}$ is crystallized on dendrite boundary at end of solidification (about 1,400 ${ }^{\circ} \mathrm{C}$ ) and finishing the solidification. And the segregated $\mathrm{Mn}$ and $\mathrm{Al}$ based oxide by the reaction with oxygen in the air.

Generally, the cooling speed of laser beam welding is $605{ }^{\circ} \mathrm{C} / \mathrm{s}$, and it is known that the cooling speed is faster than water cooling ${ }^{15)}$. So, the austenite ( $\mathrm{\gamma}$, middle of dendrite) transforms as martensite due to the rapid cooling.

Meanwhile, the phase transformation of $\mathrm{HAZ}\left(\mathrm{HAZ}_{\mathrm{BM}}\right)$ in the $\mathrm{BM}$ is as follows. It is heated as 6 zone $\left(\mathrm{A}_{1}-\mathrm{A}_{3}\right.$ temperature) in phase diagram; solution of cementite occurred in perlite, so transformed as $\mathrm{\gamma}$, and increasing $\gamma$ volume by decreasing and disappearance of ferrite when the temperature is increased. At the 5 zone temperature range, completely transformed as $\mathrm{\gamma}$, and transforms to martensite due to the rapid cooling.

Generally, the heating by laser beam is rapid heating, so the phase transformation will be shown on higher temperature than indicated in the constitutional diagram.

\section{Conclusion}

Based on the review on microstructure and hardness features of SM45C treated as surface melting hardening with Yb:YAG disc laser, the experiment result is as follows.

1) The depth of surface melting hardening layer was minimum $0.8 \mathrm{~mm}$, maximum $1.0 \mathrm{~mm}$.

2) The hardness of surface melted zone inside was $650-660 \mathrm{Hv}$ without reference to the location, and it is about $60 \mathrm{Hv}$ higher than HAZ maximum hardness (590-600 Hv). Based on the comparison of micro structure between melted zone and HAZ by EBSD, the hardness of melted zone is higher because the microstructure is minute.

3) The cooling speed of laser surface melting hardening treatment is $605{ }^{\circ} \mathrm{C} / \mathrm{s}$ (calculated with $\mathrm{t} 85$ formula) and it is faster than water cooling, so the melted zone transformed as complete martensite, and the microstructure is very minute.

4) The macrostructure inside surface melted zone has dendrite form; also there are $\mathrm{Mn}$, Si element segregate at the dendrite boundary, $\mathrm{MnS}$ at dendrite boundary, and $\mathrm{Al}$ based oxide in transgranular. However, there are no such inclusions in HAZ. Based on the interpretation of phase transformation with binary system constitutional diagram, Mns was crystallized in liquid state, and Al affiliation oxide was seems to be formed with oxygen in the air. Segregated $\mathrm{Mn}$ and $\mathrm{Si}$, and concentrated sphere shaped $\mathrm{Al}, \mathrm{S}$, and $\mathrm{O}$ is existed at the band zone in the microstructure.

\section{Acknowledgement}

This work was supported by a 2-Year Research Grant of Pusan National University

\section{References}

1. G Telasang, JD Majumdar, G Padmanabham and I Manna, Structure-property correlation in laser surface treated AISI H13 tool steel for improved mechanical properties, Materials Science and Engineering, A, 599 (2014), 255-267

2. Z. Zhang, P. Lin, D. Cong, S. Kong, H. Zhou and L. Ren, The characteristics of treated zone processed by pulsed Nd-YAG laser surface remelting on hot work steel, Optics \& Laser Technology, 64 (2014), 227-234

3. C. Wang, H. Zhou, N. Liang, C. Wang, D. Cong, C. Meng and L Ren, Mechanical properties of several laser remelting processed steels with different unit spacings, Applied Surface Science, 313 (2014), 333-340

4. C.T. Kwok, F.T. Cheng and H.C. Man, Microstructure and corrosion behavior of laser surface-melted highspeed steels, Surface \& Coatings Technology, 202 (2007), 336-348 
5. N. Yasavola, A. Abdollah-zadeh, M. Ganjali and SA Alidokht, Microstructure and mechanical behavior of pulsed laser surface melted AISI D2 cold work tool steel, Applied Surface Science, 265 (2013), 653-662

6. G. Telasang, J. Dutta Majumdar, G. Padmanabham, I. Manna, Wear and corrosion behavior of laser surface engineered AISI H13 hot working tool steel, Surface and Coatings Technology, 261 (2015), 69-78

7. Hong-Seok Choi, Byung-Min Kim and Dae-Cheol Ko, Effect of clearance and inclined angle on sheared edge and tool failure in trimming of DP980 sheet, Journal of Mechanical Science and Technology, 28(6) (2014), 23192328

8. J. Dutta Majumdar, R Galun, B.L Mordike, I Manna, Effect of laser surface melting on corrosion and wear resistance of a commercial magnesium alloy, Materials Science and Engineering, A, 361 (2003), 119-129

9. Sae-Kyoo Oh, Il-Dong Park, Tae-Eun Jeon and WonSuk Lee, Friction Welding of Dissimilar Press Punch Materials and Its Evaluation By AE, Journal of KWJS, 15 (1997), 43-53 (in Korean)

10. J. Grum and R. Sturm, Influence of laser surface melthardening conditions on residual stresses in thin plates, Surface and Coatings Technology, 100-101 (1998) 455-458
11. H.J. Shin, Y.T. Yoo, D.G. Ahn and K. Im, Laser surface hardening of S45C medium carbon steel using $\mathrm{Nd}$ :YAG laser with a continuous wave, Journal of Materials Processing Technology, 187-188 (2007), 467- 470

12. A. Kusmoko, R. Dahar, H. J. Li and S. Hadi, A Study Surface Layer and Hardness Produced by Induction Hardened S45C Steel, Applied Mechanics and Materials, 664 (2014) 43-47

13. Kwang-Hyeon Lee, Seong-Won Choi, Jung Gil Yun, Myeong-Hwan Oh, Byung Min Kim and Chung-Yun Kang, Effects of laser power on hardness and microstructure of the surface melting hardened SKD61 hot die steel using Yb:YAG disk laser, Journal of KWJS 33-3 (2015) 54-61 (in Korean)

14. Kwang-Hyeon Lee, Seong-Won Choi, Jung Gil Yun, Myeong-Hwan Oh, Byung Min Kim, and Chung-Yun Kang, Microstructure and Hardness of Yb:YAG Disc Laser Surface Overlap Melted Cold Die Steel, STD11, Journal of KWJS, 33-5 (2015) 53-60 (in Korean)

15. M Bonek, LA Dobrzański, E Hajduczek and A Klimpel, Structure and properties of laser alloyed surface layers on the hot-work tool steel, Journal of Materials Processing Technology, 175 (2006) 45-54 